\title{
Factores asociados a la no adherencia al tratamiento antirretroviral en personas con VIH/SIDA
}

Barrera-Espinoza Richar William 1,a; Gómez-Gonzales Walter Edgar 2,b; Girón-Vargas Ana 1,c; Arana-Escobar Magaly 5,d; NievaVillegas Lilia María ${ }^{1, e}$;Gamarra-Bustillos Carlos ${ }^{3, f}$; Auqui-Canchari María ${ }^{4, g}$; Zapana-Tito María* 2,6,h

\section{RESUMEN}

Objetivo: Determinar la asociación entre los factores sociales, clínicos, terapéuticos y enfermedades coexistentes con la no adherencia al tratamiento antirretroviral en personas con VIH/SIDA en el 2018.

Materiales y métodos: Estudio transversal con una muestra de 117 personas con VIH/SIDAque reciben tratamiento antirretroviral en el Hospital Daniel Alcides Carrión. Se aplicó el Cuestionario de Evaluación de la Adhesión al Tratamiento antirretroviral (CEATVIH), adaptado para su uso en Perú. El análisis se realizó con regresión logística con el programa SPSS versión 23.

Resultados: Se evidenció que el 17,10 \% de los pacientes mostró no adherencia al tratamiento, y el 82,90 \% sí se adhirió a la terapia. Las variables que tienen mayor probabilidad de asociarse a la no adherencia al tratamiento fueron el sexo $(0 R=16,175$; valor de $p=0,023$ ) y las comorbilidades relacionadas al VIH/SIDA (OR =9,556; valor de $p=0,003)$. La variable carga viral inicial $(\mathrm{OR}=0,152$; valor de $p=0,030)$ actúa como protectora, ya que existe una mayor probabilidad de adherirse al tratamiento.

Conclusiones: El sexo masculino y las comorbilidades relacionadas al VIH/SIDA están asociados a la no adherencia al tratamiento antirretroviral. La variable carga viral inicial actúa como un factor protector para la adherencia al tratamiento.

Palabras clave: Adherencia al tratamiento; Terapia antirretroviral; VIH; Síndrome de inmunodeficiencia adquirida (Fuente: DeCS BIREME).

\section{Factors associated with nonadherence to antiretroviral therapy in people with HIV/AIDS}

\section{ABSTRACT}

Objective: To determine the association that social, clinical and therapeutic factors and coexisting diseases have with nonadherence to antiretroviral therapy in people with HIV/AIDS in 2018.

Materials and methods: A cross-sectional study conducted with a sample of 117 people with HIV/AIDS on antiretroviral therapy at the Hospital Daniel Alcides Carrión. The Questionnaire for the Assessment of Adherence to Antiretroviral Therapy (CEAT-VIH) adapted for use in Peru was administered. The IBM SPSS Statistics V23 and logistic regression were used for the statistical analysis. Results: Seventeen point one zero percent (17.10\%) of the patients did not adhere to the therapy and $82.90 \%$ adhered to their prescribed treatment. The variables most likely to predict nonadherence were sex $(\mathrm{OR}=16.175, p$ value $=0.023)$ and HIV/AIDSrelated comorbidities $(O R=9.556, p$ value $=0.003)$. The initial viral load variable $(O R=0.152, p$ value $=0.030)$ acts as a protector because there is a greater probability of treatment adherence.

Conclusions: Male sex and HIV/AIDS-related comorbidities are associated with the nonadherence to antiretroviral therapy. The initial viral load variable acts as a protector for treatment adherence.

Keywords: Treatment adherence and compliance; Antiretroviral therapy; HIV; Acquired immunodeficiency syndrome (Source: MeSH NLM).

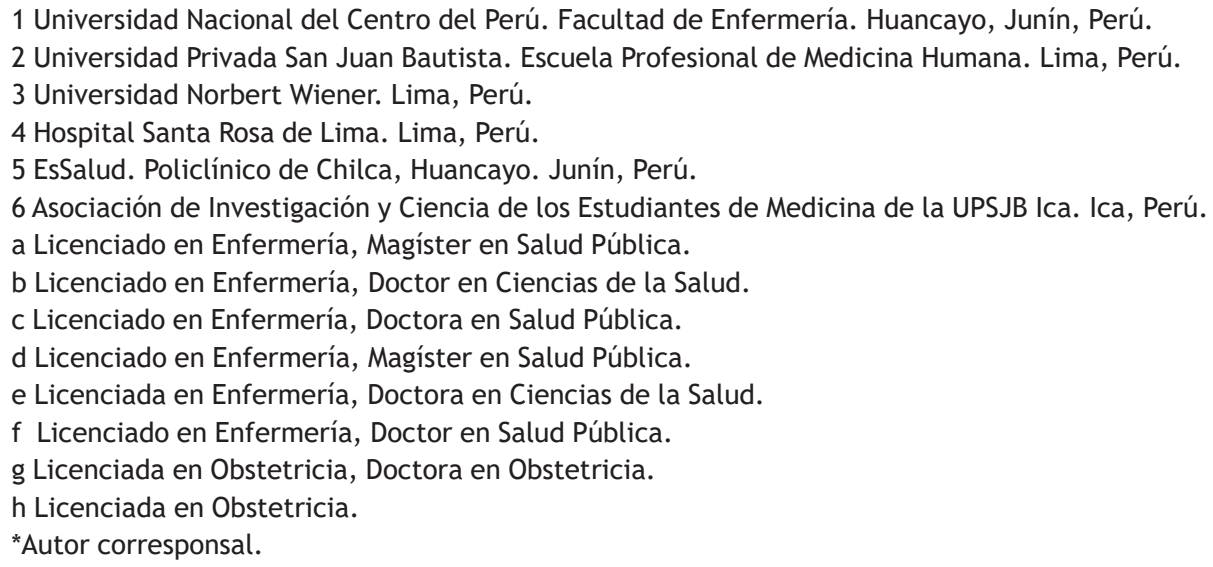


Barrera-Espinoza Richar William; Gómez-Gonzales Walter Edgar; Girón-Vargas Ana; Arana-Escobar Magaly; Nieva-Villegas Lilia María;Gamarra-Bustillos

Carlos; Auqui-Canchari María; Zapana-Tito María

\section{INTRODUCCIÓN}

El síndrome de inmunodeficiencia adquirida (SIDA) es una manifestación clínica avanzada de la infección por el virus de la inmunodeficiencia humana (VIH). En esta etapa la inmunodeficiencia es grave, lo que da lugar a infecciones y neoplasias asociadas que pueden causar la muerte ${ }^{(1)}$.

En la actualidad, el número de personas con VIH/SIDA se ha incrementado, por lo que se le considera como un problema de salud pública (2). Estos pacientes han mejorado con los fármacos antirretrovirales de gran actividad (TARGA) y las combinaciones de terapias que ayudan a reducir la transmisión del virus, elevar la esperanza de supervivencia y mejorar la calidad de vida de los portadores. Existe una amplia evidencia de que los pacientes han revolucionado sus horizontes con este tratamiento.

El manejo de las personas infectadas con VIH/SIDA con el tratamiento antirretroviral es de largo plazo. La ingesta prolongada de medicamentos ocasiona que se incumpla el tratamiento y, en consecuencia, la terapia se hace ineficiente, el virus se vuelve resistente y se incrementa el número de casos de $\mathrm{VIH}{ }^{(3)}$. Para catalogar como correcta una adherencia al tratamiento, el índice debe ser mayor de $95 \%$, esto significa que el paciente no puede dejar de tomar (o hacerlo tardíamente) más de cinco pastillas por mes, o dosis tomadas de manera apropiada ${ }^{(4)}$.

En el 2004, en el Perú se implementó el Programa de Tratamiento Antirretroviral de Gran Amplitud (TARGA) ${ }^{(5)}$ con el objetivo de proporcionar antirretrovirales gratuitos. Según el Ministerio de Salud, en el 2020 el número de personas que vivirán con el VIH es de 132657 , de las cuales 45089 estarán en el estadio de sida; el mayor número de enfermos serán hombres y mujeres de 15 a 49 años (6). En el mundo, la magnitud del grado de no adhesión a los tratamientos de enfermedades crónicas varía de $25 \%$ a $50 \%$, y es mucho mayor en los países en vías de desarrollo, donde se asocia a la pobreza y a menor acceso a los servicios sanitarios ${ }^{(7)}$.

Diferentes estudios señalan elementos vinculados a la adherencia: variables individuales, características del tratamiento, características de la infección por VIH/SIDA y relación con los servicios de salud y apoyo social, lo que confirma el carácter heterogéneo y regional de los factores asociados con la adherencia (1). Otras investigaciones encontraron factores como edad menor de 40 años, nivel socioeconómico bajo, tomar más de cuatro pastillas diarias, y se perciben barreras para el tratamiento de las mujeres con una pareja o hijos diagnosticados con VIH/SIDA ${ }^{(8)}$. Del mismo modo, otro estudio internacional halló que la baja escolaridad, el vínculo de pareja inestable, la disfunción familiar, el tiempo de tratamiento mayor de 5 años y la aparición de reacciones adversas como factores de riesgo de mala adherencia terapéutica a los antirretrovirales ${ }^{(9)}$.

La terapia antirretroviral es la piedra angular de la prevención y el tratamiento de la infección por el $\mathrm{VIH}{ }^{(10)}$, es una importante estrategia para las personas con VIH/SIDA, de tal manera que es fundamental que la persona, de manera ordenada y regular, ingiera los retrovirales. Tan solo en la región Junín viven 705 personas con VIH y 143 con sida; en el 2018, se notificaron 57 casos de VIH y 34 casos de sida al primer semestre, estos datos la ubican como la quinta región con mayor índice de casos ${ }^{(11)}$. En la misma región, se reportaron 195 casos de VIH en el 2019, y 105 en el $2020^{\text {(12). }}$

Por tal motivo, es necesario establecer cuáles son los factores asociados a la adherencia al TARGA. En ese sentido, el objetivo de estudio fue determinar los factores asociados a la adherencia al tratamiento antirretroviral de gran actividad en personas con VIH/SIDA en el Hospital Daniel Alcides Carrión de Huancayo en 2018.

\section{MATERIALES Y MÉTODOS}

\section{Diseño y población de estudio}

Investigación correlacional múltiple. Los datos se recopilaron con el Cuestionario de Evaluación de la Adhesión al Tratamiento Antirretroviral (CEAT-VIH), adaptado para su uso en Perú, que evalúa el cumplimiento del tratamiento en la última semana. El cuestionario consta de 20 ítems que evalúan cumplimiento del tratamiento, antecedentes de la falta de adherencia, interacción con el médico, creencias del paciente y estrategias para mejorar la adherencia. La puntuación total oscila entre 17 y 89 . El baremo para el instrumento fue adherencia (17-72) y no adherencia (73-87).

La población estuvo conformada por 540 personas con $\mathrm{VIH} / \mathrm{SIDA}$ que reciben tratamiento antirretroviral de gran amplitud. La muestra incluyó a 117 personas, para ello se tuvo en cuenta el número de variables independientes que se consideraron en la investigación. Los casos se seleccionaron a través del muestreo no aleatorio simple, teniendo como criterio de inclusión la participación voluntaria de las personas. Los investigadores aplicaron el instrumento de manera anónima e individual a las personas durante la entrega de los medicamentos antirretrovirales. Los datos se recogieron en el último trimestre del año 2018. La base de datos se identificó mediante el número de historia clínica. El instrumento de adherencia tiene una confiabilidad de alfa de Cronbach de 0,706 y el de factores 0,662.

\section{Variables y mediciones}

La variable dependiente fue la no adherencia al tratamiento antirretroviral. Como variables independientes, figuran los actores asociados (edad, sexo, grado de instrucción, estado civil, consumo de drogas, consumo de alcohol, vía de contagio, carga viral inicial, carga viral final, CD4 inicial, CD4 último, años de tratamiento, RAM y comorbilidades asociadas a VIH/SIDA). 


\section{Análisis estadístico}

El análisis estadístico se realizó mediante regresión logística con 16 variables independientes y la variable dependiente dicotómica, con un nivel de significancia del $5 \%$. Los datos se procesaron en el programa estadístico SPSS-23.

\section{Consideraciones éticas}

El estudio fue aprobado por la Facultad de Enfermería de la Universidad Nacional del Centro del Perú. En la ejecución del estudio se han respetado los principios de la bioética de la investigación en seres humanos, en el marco de la Declaración de Helsinki y las modificatorias Edimburgo. Se guardó la confidencialidad de los datos.

\section{RESULTADOS}

Los resultados se presentan utilizando la estadística descriptiva. Las tablas simples se usaron para medir la adherencia al TARGA y describir los factores asociados a la adherencia.

La tabla 1 muestra que la edad de los pacientes varió entre los 18 y 69 años (promedio de 39,28 $\pm 11,671$ ). El 70,90 \% (83) son hombres y 29,10 \% (34), mujeres. El
$59,80 \%$ (70 pacientes) tenía instrucción básica; y el 40,20\% (47), educación superior. El 54,70 \% (64 personas) viven sin pareja, y el 45,30 \% (53) tienen pareja. Solo una paciente consume droga $(0,90 \%)$. La ingesta de alcohol es un hábito en 38 pacientes (32,50\%), mientras que el 67,50\% (79 participantes) no consume bebidas alcohólicas. Respecto al contagio, en 110 casos tuvo contagio por la vía sexual $(94,00 \%)$ y 7 pacientes $(6,00 \%)$ se contagiaron por vía no sexual. En 102 pacientes $(87,20 \%$ ), la carga viral inicial fue mayor de 1000 copias $/ \mathrm{ml}$; y en 15 personas $(12,80 \%)$, menor de 1000 copias $/ \mathrm{ml}$. El 13,70 \% (16 casos) tiene una última carga viral mayor a 1000 copias $/ \mathrm{ml}$, y el $86,30 \%$ (101 casos) tiene menos de 1000 copias $/ \mathrm{ml}$.

El $36,80 \%$ (43) tiene linfocitos CD4 detectables al inicio; y en el $63,20 \%$ (74) de los casos los CD4 no se podían detectar. En el último recuento, los linfocitos CD4 fueron detectables en 87 pacientes (74,40\%), e indetectables en 30 personas $(25,60 \%)$. El 55,60\% (65) tiene menos de 5 años de tratamiento, y el $44,40 \%$ (52), más de 5 años. Seis pacientes tuvieron una reacción adversa al tratamiento; los demás no mostraron reacción adversa. El 28,20\% (33) presenta comorbilidades asociadas a VIH/SIDA, y $71,80 \%$ (84), no presenta.

Tabla 1. Características de las personas infectadas con VIH/SIDA

\begin{tabular}{lrr} 
Características & Número & Porcentaje (\%) \\
\hline Edad & & \\
Menos de 60 años & 109 & 93,20 \\
Más de 60 años & 8 & 6,80 \\
Sexo & & \\
Hombre & 83 & 70,90 \\
Mujer & 34 & 29,10 \\
Grado de instrucción & & \\
Básico & 70 & 59,80 \\
Superior & 47 & 40,20 \\
Estado civil & & \\
Soltero (a) & 64 & 54,7 \\
Casado (a) & 9 & 7,7 \\
Unión libre & 22 & 18,8 \\
Separado & 15 & 12,8 \\
Viudo & 6 & 5,1 \\
Divorciado & 1 & 0,9 \\
Ocupación & & \\
Trabaja en casa & 9 & 7,7 \\
Estudiante & 6 & 5,1 \\
Trabajo dependiente & 35 & 29,9 \\
Trabajo independiente & 49 & 41,9 \\
Trabajo eventual & 18 & 15,4 \\
- & &
\end{tabular}




\begin{tabular}{|c|c|c|}
\hline Características & Número & Porcentaje (\%) \\
\hline \multicolumn{3}{|c|}{ Consumo de droga } \\
\hline Sí & 1 & 0,90 \\
\hline No & 116 & 99,10 \\
\hline \multicolumn{3}{|c|}{ Consumo de alcohol } \\
\hline Sí & 38 & 32,50 \\
\hline No & 79 & 67,50 \\
\hline \multicolumn{3}{|c|}{ Tiempo de diagnóstico } \\
\hline Menos de 5 años & 63 & 53,8 \\
\hline De 5 años a más & 54 & 46,2 \\
\hline \multicolumn{3}{|l|}{ Vía de contagio } \\
\hline Sexual & 110 & 94,00 \\
\hline No sexual & 7 & 6,00 \\
\hline \multicolumn{3}{|l|}{ Carga viral inicial } \\
\hline Más de 1000 & 102 & 87,20 \\
\hline Menos de 1000 & 15 & 12,80 \\
\hline \multicolumn{3}{|l|}{ Carga viral última } \\
\hline Más de 1000 & 16 & 13,70 \\
\hline Menos de 1000 & 101 & 86,30 \\
\hline \multicolumn{3}{|l|}{ CD4 inicial } \\
\hline Detectable & 43 & 36,80 \\
\hline No detectable & 74 & 63,20 \\
\hline \multicolumn{3}{|l|}{ CD4 último } \\
\hline Detectable & 87 & 74,40 \\
\hline No detectable & 30 & 25,60 \\
\hline \multicolumn{3}{|c|}{ Años de tratamiento } \\
\hline Menos de 5 años & 65 & 55,60 \\
\hline De 5 a más años & 52 & 44,40 \\
\hline \multicolumn{3}{|l|}{ RAM } \\
\hline Sí & 6 & 5,10 \\
\hline No & 111 & 94,90 \\
\hline \multicolumn{3}{|c|}{ Comorbilidades asociadas a VIH/SIDA } \\
\hline Sí & 33 & 28,20 \\
\hline No & 84 & 71,80 \\
\hline
\end{tabular}

En la tabla 2 se muestra que el $19,70 \%$ presenta no adherencia, y el $80,30 \%$ sí muestra adherencia al tratamiento antirretroviral.

Tabla 2. Adherencia al tratamiento antirretroviral de las personas con VIH/SIDA

\begin{tabular}{ccc} 
Adherencia a tratamiento & Frecuencia & Porcentaje \\
\hline Sí & 23 & 19,70 \\
No & 94 & 80,30 \\
Total & 117 & 100,00 \\
\hline
\end{tabular}


La tabla 3 muestra las variables que están asociadas a la no adherencia. Según el análisis multivariado, el sexo masculino y las comorbilidades relacionadas al $\mathrm{VIH}$ se encuentran asociadas a la no adherencia al tratamiento antirretroviral en personas con VIH/SIDA. Respecto al sexo, los hombres tienen mayor probabilidad de no adherirse al tratamiento, al igual que las personas que presentan comorbilidades relacionadas al VIH/SIDA. La variable carga viral inicial actúa como protectora debido a que hay mayor probabilidad de adherirse al tratamiento cuando presenta la carga viral mayor a 1000 copias/ml.

Tabla 3. Factores asociados a la no adherencia al tratamiento antirretroviral

\begin{tabular}{|c|c|c|c|c|c|c|c|}
\hline & & B & $\begin{array}{l}\text { Error } \\
\text { estándar }\end{array}$ & Wald & GI & Sig. & $\operatorname{Exp}(B)$ \\
\hline \multirow[t]{17}{*}{ Paso 1a } & Edad de los pacientes &,- 056 & ,035 & 2,580 & 1 & ,108 & ,945 \\
\hline & Sexo de los pacientes & 2,783 & 1,225 & 5,167 & 1 & ,023 & 16,175 \\
\hline & Grado de instrucción & ,952 &, 580 & 2,695 & 1 & ,101 & 2,590 \\
\hline & Estado civil &,- 218 & ,309 & ,496 & 1 & ,481 & ,804 \\
\hline & Ocupación del paciente &, 400 & ,359 & 1,242 & 1 & ,265 & 1,492 \\
\hline & Consume drogas & $-16,080$ & 40193,049 &, 000 & 1 & 1,000 & ,000 \\
\hline & Consume alcohol & 1,065 & ,711 & 2,240 & 1 & ,134 & 2,900 \\
\hline & Años de diagnóstico & 1,664 & 1,079 & 2,379 & 1 & ,123 & 5,283 \\
\hline & Vía de contagio & $-2,093$ & 1,172 & 3,190 & 1 & ,074 & ,123 \\
\hline & Carga viral inicial & $-1,886$ &, 869 & 4,712 & 1 & ,030 & ,152 \\
\hline & Carga viral ultima & $-40,216$ & 9237,818 &, 000 & 1 & ,997 & ,000 \\
\hline & CD4 inicial & ,960 &, 881 & 1,188 & 1 & ,276 & 2,611 \\
\hline & CD4 última &,- 378 &, 938 & ,162 & 1 & ,687 & ,685 \\
\hline & Años de tratamiento & ,113 & 1,020 & ,012 & 1 & ,912 & 1,119 \\
\hline & RAM reportada por el paciente & 21,974 & 6043,395 & ,000 & 1 & ,997 & 3494522443,203 \\
\hline & Comorbilidades asociadas a VIH & 2,257 & ,773 & 8,532 & 1 & ,003 & 9,556 \\
\hline & Constante & 147,873 & 85123,148 &, 000 & 1 & ,999 & $1,662 E+64$ \\
\hline
\end{tabular}

\section{DISCUSIÓN}

En el Perú, hasta enero de 2021 se han notificado al sistema de vigilancia 137839 casos de personas infectadas por $\mathrm{VIH}$; de ellos, 45703 están en el estadio sida. Para el año 2020, la relación hombre/mujer en pacientes con VIH fue de 3 a 1 , es decir, por cada mujer había 3 hombres infectados; esta relación es menor a la de 2019 , que fue de 4 a 1 . Respecto a la relación hombre/mujer en personas en estadio sida, fue 3,2 en el $2020^{(6)}$.

El tratamiento antirretroviral administrado a personas infectadas por el VIH busca reducir la replicación de ARN en la sangre y normalizar el recuento de CD4, con este fin se usan fármacos combinados que disminuyen la probabilidad de resistencia a los medicamentos. Los beneficios del tratamiento son reducir la morbilidad y mortalidad y mejorar la calidad de vida de las personas infectadas (13). Lo importante es promover la adherencia, definida como el grado en el que la conducta de un paciente cambia, en relación con la toma de medicación, el seguimiento de una dieta o la modificación de hábitos de vida ${ }^{(14)}$.Los resultados indican que el $19,70 \%$ no se adhiere al tratamiento antirretroviral, resultado que es similar al $14 \%$ de no adherencia encontrado en Paraguay ${ }^{(15)}$ y el $17,4 \%$ de no adherencia encontrado en Perú por Orellana (16). En Cuba se halló que el 29,5\% no presenta adherencia al tratamiento, y entre los factores determinantes de la no adherencia están el grado académico, edad, ingresos, así como factores personales, interpersonales (como el apoyo sociofamiliar, estigma percibido, autoestima) y factores relativos a la enfermedad y tratamiento ${ }^{(17)}$.

Según el análisis multivariado, los resultados indican que el sexo masculino y las comorbilidades relacionadas al VIH se encuentran asociados a la no adherencia: 16 veces más probabilidades en los hombres y 9 veces más en quienes padecen comorbilidades relacionadas al $\mathrm{VIH}$. La carga viral inicial funciona como una variable protectora, ya que las personas que tienen una carga viral mayor a 1000 copias $/ \mathrm{ml}$ tienen mayor probabilidad de adherirse al tratamiento. Los resultados difieren de otras investigaciones como la de Pérez, que señala como variables asociadas a no adherencia al consumo de cocaína en los últimos 6 meses, no estar totalmente seguros de 
poder tomar correctamente los tratamientos prescritos y no tener prescritos la combinación de análogos zidovudina (AZT) y lamivudina ${ }^{(9)}$. El estudio de Urizar detectó que las variables asociadas a no adherencia son la presencia de efectos adversos y el olvido de la toma de la medicación durante el fin de semana ${ }^{(15)}$. Los hallazgos que coinciden en las investigaciones son el tiempo de tratamiento y la presencia de reacciones adversas.

La investigación ha demostrado que existe una relación entre la variable sexo masculino y la no adherencia al tratamiento, lo que probaría lo señalado por otros estudios respecto a que las mujeres muestran una mejor adherencia a los tratamientos de enfermedades crónicas. La razón de esta diferencia sería la mayor responsabilidad de las mujeres, de acuerdo con lo reportado por investigaciones sobre la responsabilidad laboral (15). Las comorbilidades son otra variable asociada a la no adherencia al tratamiento y que en el caso de los portadores de $\mathrm{VIH}$ son las dislipidemias y las infecciones de transmisión sexual ${ }^{18)}$. Estos padecimientos precisan de un tratamiento para su control, ocasionando el consumo de otros medicamentos, lo que puede confundir al paciente y, si no tiene un orden adecuado, hacer que olvide las terapias.

De acuerdo con los resultados, es fundamental señalar que la variable carga viral inicial funciona como factor protector debido a que la probabilidad de adherirse al tratamiento es mayor si la persona tiene una carga viral inicial mayor a 1000 copias $/ \mathrm{mL}$. Esto indica que un valor alto de carga viral hace que los pacientes consideren importante cumplir con la terapia. En este sentido, la educación que brinda el personal de salud influye en la conducta del paciente al destacar el papel del tratamiento para disminuir la carga viral. Es menester aclarar que para lograr una óptima adherencia al tratamiento no basta con la entrega del fármaco para que el paciente lo autoadministre, sino que interviene también el incentivo educacional como un reto de la actividad asistencial. Se concluye que el sexo masculino y las comorbilidades relacionadas al $\mathrm{VIH}$ se encuentran asociadas a la no adherencia al tratamiento antirretroviral en personas con VIH/SIDA, y la variable carga viral inicial actúa como protector, ya que presenta una mayor probabilidad de adherencia al tratamiento. Por ello, se recomienda a los profesionales de la salud fortalecer el componente educativo durante el diagnóstico y el inicio del tratamiento antirretroviral con la finalidad de promover la adherencia al tratamiento en personas con $\mathrm{VIH}$. Como limitaciones del estudio señalamos que los resultados no se pueden inferir debido a que el tamaño de muestra es pequeño y que en los antecedentes se utilizaron diferentes instrumentos para medir la variable adherencia al tratamiento antirretroviral.

Contribuciones de los autores: Richar William Barrera Espinoza: conceptualización, metodología, el análisis formal, investigación, curación de datos, la redacciónpreparación del borrador original y la redacción, revisión y edición.

Walter Edgar Gómez Gonzales, Carlos Gamarra Bustillos, María Auqui Canchari y María Teresa Zapana Tito: curación de datos, la redacción-preparación del borrador original y la redacción-revisión y edición.

Ana Girón Vargas: análisis formal e investigación.

Magaly Arana Escobar: conceptualización, la metodología, los recursos y la redacción-preparación del borrador original.

Lilia María Nieva Villegas: validación, el análisis formal, la curación de datos, la redacción-preparación del borrador original y la redacción-revisión y edición.

Fuentes de financiamiento: El artículo ha sido financiado por los autores.

Conflictos de interés: Los autores declaran no tener conflictos de interés.

\section{REFERENCIAS BIBLIOGRÁFICAS}

1. Carvalho PP, Barroso SM, Coelho HC, Penaforte FRO. Factors associated with antiretroviral therapy adherence in adults: an integrative review of literature. Cien Saude Colet. 2019; 24(7): 2543-55.

2. Murillo JMA, Vera DRZ, Vera CIA, Zambrano VPP. Perfil Epidemiológico del VIH en Latinoamérica. RECIMUNDO Rev Científica Investig El Conoc. 2019; 3(1): 232-58.

3. da Silva RAR, Costa MMN, Neto VLS, da Silva BCO, Costa CS, de Andrade IFC. Noncompliance in people living with HIV: accuracy of defining characteristics of the nursing diagnosis. Rev Latino-Am Enfermagem. 2017; 25: e2940.

4. Lamillar Taipe E. Adherencia al Tratamiento Antirretroviral en Pacientes del Programa de Control de Infecciones de Transmisión Sexual y SIDA del Hospital Nacional "Hipólito Unanue", Lima, Perú, 2018 [Tesis]. Lima: Universidad Ricardo Palma. Facultad de Medicina; 2019.

5. Canto YE, Díaz Gervasi GMD, Alvirio LAM. Impacto del Programa TARGA en la disminución de casos de sida en el sistema de salud peruano, 1983-2018. Rev Panam Salud Pública. 2020; 44: e27.

6. Ministerio de Salud. Situación epidemiológica del VIH-Sida en el Perú [Internet]. MINSA: Lima; 2021. Disponible en: https://www.dge.gob. pe/portal/docs/vigilancia/vih/Boletin_2021/febrero.pdf

7. Frómeta LV, Alfonso LM, Nariño IP. Adherencia terapéutica y apoyo social percibido en personas que viven con VIH/sida. Rev Cubana Salud Pública. 2015; 41(4).

8. Arévalo MTV, Gutiérrez OLG, Marían HFM, Quintero JG, Bolívar ICT. Factores relacionados con la adherencia al tratamiento farmacológico para el VIH/SIDA. Rev Salud Uninorte. 2013; 29(1): 83-95.

9. Bastán JEP, Castaño LFV. Factores asociados a la no adherencia terapéutica a los antirretrovirales en personas con $\mathrm{VIH} /$ sida. Rev Cubana Med Trop. 2020; 72(2): e499.

10. Saag MS, Benson CA, Gandhi RT, Hoy JF, Landovitz RJ, Mugavero MJ, et al. Antiretroviral Drugs for Treatment and Prevention of HIV Infection in Adults: 2018 Recommendations of the International Antiviral Society-USA Panel. JAMA. 2018; 320(4): 379-96. 
11. Ministerio de Salud. Situación epidemiológica del VIH-Sida en el Perú [Internet]. MINSA: Lima; 2018. Disponible en: http://www.dge.gob. pe/portal/docs/vigilancia/vih/Boletin_2018/junio.pdf

12. Centro Nacional de Epidemiología, Prevención y Control de Enfermedades, Ministerio de Salud. Sala situacional VIH/ SIDA, CDC Perú [Internet]. Tableau Software. CDC, MINSA: Lima; 2021. Disponible en: https://public.tableau.com/views/ TABLEUVIH_SIDAfinal_15601794344210/fig1_Curvavih?:emb ed=y\&: showVizHome=no\&: host_url=https\%3A\%2F\%2Fpublic. tableau.com\%2F\&:embed_code_version=3\&: tabs=no\&: toolbar=yes\&: animate_transition=yes\&:display_static image=no\&:display_spinner=no\&:display_overlay=yes\&:display_ count $=$ yes\&:loadOrderID $=0$

13. Organización Panamericana de la Salud, Organización Mundial de la Salud. Terapia Antirretroviral [Internet]. 2020. Disponible en: https://www.paho.org/es/temas/terapia-antirretroviral

14. Cerda JOC, Herrera DS, Miranda OAR, Legaspi JMO. Adherencia terapéutica: un problema de atención médica. Acta Médica Grupo Ángeles. 2018; 16(3): 226-32.

15. Urizar CA, Jarolin-Montiel M, Ayala-Servin N, Centurión-Wenninge C, Montiel-Garcete D. Factores asociados a la no adhrencia del tratamiento antirretroviral en pacientes con $\mathrm{VIH}$ en un hospital de Paraguay. Rev Cient Cienc Méd. 2020; 23(2): 166-74.

16. Orellana-Zanabria G, Morales-Rezza E. Factores asociados a la adherencia al TARGA, en pacientes con VIH/SIDA en el hospital central PNP "Luis N. Sáenz" en los meses de octubre a diciembre del 2015. Rev Fac Med Hum. 2019; 19(1): 46-54.

17. Peña SS, Bravo MdMP, Tomás MAC, Martínez PA, Guillen CP, JiménezRuiz I. Factores relacionados con la adherencia al tratamiento antirretroviral en mujeres con VIH: Un estudio mixto con diseño secuencial. Enferm Glob. 2021; 20(62): 1-34.

18. Díaz Agudelo TC. Comorbilidad de pacientes que viven con VIH y pertenecen al programa de atención integral de una IPS de Bucaramanga [Tesis]. Bucaramanga: Universidad De Santander UDES. Facultad de Ciencias Económicas, Administrativas y Contables; 2019.

\section{Correspondencia:}

María Teresa Zapana-Tito.

Dirección: Urbanización las Mercedes B-06. Ica, Perú.

Teléfono: +051955164010

Correo electrónico: maria.zapana@upsjb.edu.pe

Recibido: 01 de mayo de 2021.

Evaluado: 03 de agosto de 2021.

Aprobado: 02 de octubre de 2021.

(c) La revista. Publicado por Universidad de San Martín de Porres, Perú. (c) Br Licencia de Creative Commons Artículo en acceso abierto bajo términos de Licencia Creative Commons Atribución 4.0 Internacional. (http://creativecommons.org/licenses/by/4.0/)

ORCID iDs

Barrera-Espinoza Richar William Gómez-Gonzales Walter Edgar

Girón- Vargas Ana

Arana - Escobar Magaly

Nieva -Villegas Lilia María

Gamarra-Bustillos Carlos

Auqui-Canchari María

Zapana-Tito María
(1) https: / / orcid.org/0000-0002-6637-1109

(1) https: / / orcid.org/0000-0003-0706-7614

(1) https: / / orcid.org/0000-0002-7022-1904

(1) https: / / orcid.org/0000-0002-3858-2080

(1) https: / / orcid.org/0000-0001-9889-6332

(1) https: / / orcid.org/0000-0003-0487-9406

(i) https: / / orcid.org/0000-0003-3251-9096

그 https: / / orcid.org/0000-0003-3374-7349 